\title{
Correlation of macrophage migration inhibitory factor gene polymorphism with the risk of early-stage cervical cancer and lymphatic metastasis
}

\author{
SUHUI WU, JUNFANG LIAN, HUIJUAN TAO, HAIXIA SHANG and LI ZHANG \\ Department of Gynecology, the First Hospital, Shanxi Medical University, Taiyuan 030001, P.R. China
}

Received March 1, 2011; Accepted July 21, 2011

DOI: $10.3892 / \mathrm{ol} .2011 .409$

\begin{abstract}
Associations of functional single nucleotide polymorphisms in MIF-173G/C with early stage cervical cancer were investigated in a hospital-based case-control study on 250 patients with cervical cancer prior to surgery (including 49 cases with and 201 cases without lymphatic metastasis) and 147 healthy controls. The polymorphism was assessed using restriction fragment length polymorphism polymerase chain reaction, and the MIF serum concentration was examined using the enzyme-linked immunosorbent assay to analyze the correlation between the polymorphism and the MIF serum concentration. Carriers of the variant $\mathrm{C}$ allele in MIF-173 were at a significantly higher risk of cervical cancer compared to carriers of the wild-type allele $(\mathrm{aOR}=1.508 ; 95 \%$ CI, 1.128-2.016, $\mathrm{p}=0.05)$. The GC and CC genotypes may be the causative factors for cervical cancer $(\mathrm{aOR}=1.851 ; 95 \%$ $\mathrm{CI}, 1.132-3.027, \mathrm{p}=0.013)$. Individuals with the $\mathrm{GC}+\mathrm{CC}$ genotype and $\mathrm{C}$ allele at the $\mathrm{MIF}-173 \mathrm{G} / \mathrm{C}$ site were at a significantly higher risk of cervical cancer and lymphatic metastasis. The risk of lymphatic metastasis in early stage cervical cancer was increased more than 1.6 times in patients with the $\mathrm{CC}$ and $\mathrm{GC}$ genotypes compared with those with the GG genotype. The genotype distribution and allele frequency of MIF-173G/C were statistically significant in the well-, moderately and poorly differentiated groups $(\mathrm{P}<0.05)$. Compared to the $\mathrm{GG}$ genotype and $\mathrm{G}$ allele, patients with $\mathrm{GC}$ and $\mathrm{CC}$ genotypes and $\mathrm{C}$ allele exhibited a lower degree of differentiation and a higher degree of malignancy. A significant difference was observed in MIF serum concentrations among the various subgroups $(\mathrm{P}<0.05)$. The early cervical cancer, lymphatic metastasis and poorly differentiated groups exhibited higher MIF levels in serum. Moreover, patients with the CC genotype exhibited higher MIF serum concentration, which could increase the risk of
\end{abstract}

Correspondence to: Dr Suhui Wu, Department of Gynecology, the First Hospital, Shanxi Medical University, Taiyuan 030001, P.R. China

E-mail: shwu1215@163.com

Key words: cervical cancer, MIF, gene polymorphisms, restriction fragment length polymorphism polymerase chain reaction, enzymelinked immunosorbent assay early stage cervical cancer and lymphatic metastasis. The results presented in this study provide the first evidence that the genetic polymorphism MIF-173 is associated with cervical cancer in humans. Detection of MIF serum concentration and genotyping may be used as biomarkers for early diagnosis and therapy for cervical cancer.

\section{Introduction}

Cervical cancer is the second most common cancer among women worldwide, with an estimated 493,000 new cases and 274,000 deaths in the year 2002, of which $83 \%$ occurred in developing countries (1). Although a substantial decrease in both incidence and mortality rates has been observed in the past 30 years, with the age of patients becoming increasingly younger (2,3). More than 100,000 cervical cancer cases are diagnosed in China each year, and lymph node metastasis is the most common form of metastasis in the early stages of cervical cancer (4). Shanxi Province has the highest incidence of cervical cancer in China. The etiology of cervical cancer is not entirely understood: human papillomavirus (HPV) infection has become globally accepted as one of the main causes (5) and the correlation between inflammation and cancer has been the subject of extensive investigation in China and worldwide. The majority of HPV infections are cleared without further consequences for the host: however, untreated infection with certain high-risk HPV types may cause high-grade cervical intraepithelial neoplasia (grades II-III) and cervical cancer (6). A number of studies indicated that the inflammatory factor plays a crucial role in the occurrence and development of cancer (7-10). This observation is supported by the epidemiological relationship between chronic or recurrent local inflammation and cancer development, such as the association between Barrett's esophagus and esophageal cancer, Helicobacter gastritis and gastric cancer, chronic pancreatitis and pancreatic cancer, cirrhosis and hepatic cancer, and inflammatory bowel disease and colon carcinoma (7). Current research has predominantly focused on single-nucleotide polymorphisms (SNPs). Studies have found that the SNPs of oncogenes were associated with tumor metastasis and malignant potential. The SNP of the coding or promoter regions is of significance in medical research and may be used as a tumor marker in the early diagnosis and prevention of cancer. 
Macrophage migration inhibitory factor (MIF) is a lymphocyte factor whose main role is to restrain the mobility of macrophages, promoting macrophage accumulation in Type IV allergic reactions (11). It is also a type of inflammatory cell factor with extensive roles and a high expression in certain tumor tissues. MIF is involved in tumor cell proliferation and differentiation, promotes tumor invasion and metastasis (12) and is capable of increasing the susceptibility to diseases such as lung (13) and breast cancer (14), gastric carcinogenesis (15) and prostate cancer (16). The human MIF gene situated on chromosome 22 (q11.23) has a 2-kb structure with three exons and two introns (17). A ' $G$ ' to ' $C$ ' SNP at the -173 position plays a significant role in gastric carcinogenesis (15) and prostate cancer (16) where, in conjunction with $\mathrm{C}$ alleles, it is associated with increased genetic predisposition and is significantly associated with tumor invasion and metastasis and malignant potential. However, the role and effect of MIF-173G/C in early cervical cancer has yet to be investigated.

We hypothesized that MIF-173G/C polymorphisms are significant in the development and invasiveness of cervical cancer. The MIF-173 G/C genotype was examined in primary cervical cancer patients and healthy controls in the Shanxi Province to investigate whether there is an association between MIF gene polymorphism at -173 and the incidence of cervical cancer, risk of lymph node metastasis and degree of malignancy. We also examined the pathogenesis of malignant transformation of tumor cells at the molecular level. Additionally, we examined the serum MIF concentrations of primary cervical cancer patient and healthy control groups, patients with and without lymph node metastases and patient groups with various degrees of malignancy, and investigated the relationship between the MIF-173G/C polymorphism and MIF serum concentrations of primary cervical cancer patients. We postulate that MIF is a tumor marker for early diagnosis and prevention of cervical cancer.

\section{Materials and methods}

Study population. Using a case-control study, 250 newly diagnosed, histologically confirmed Ib-IIa period cervical cancer patients were consecutively recruited between May 2009 and December 2009 from the First Affiliated Hospital of Shanxi Medical University and the Shanxi Tumor Hospital (treated without chemotherapy), with an average age of $49.08 \pm 9.405$ years. The non-cancer controls $(n=147)$ were composed of outpatients who visited the First Affiliated Hospital of Shanxi Medical University to participate in a routine cervical cancer screening program. These subjects did not exhibit any history of cervical disease nor present any evidence of cervical pathology. Additionally, patients with active infectious diseases, chronic inflammatory diseases, or histologically undetermined cervical abnormalities were excluded from this study to limit selection bias. The average age was $47.99 \pm 10.750$ and the average ages in the two groups compared were not statistically significant. The case and control subjects were born in the Shanxi province, China.

Genotyping. Genomic DNA was extracted from EDTAanticoagulated peripheral blood samples, according to the salting-out method. Polymerase chain reaction (PCR) amplifi- cation of the region of interest was carried out with forward (5'-ACTAAGAAAGACCCGAGGC-3') and reverse 5'-GGGG CACGTTGGTGTTTAC-3') primers. PCR reagents in $25 \mu \mathrm{l}$ reaction volume contained $100 \mathrm{ng}$ of genomic DNA, $0.5 \mu \mathrm{l}$ of each oligonucleotide primer $(10 \mu \mathrm{M}), 2.5 \mu 1$ 10X PCR buffer, $2.0 \mu \mathrm{l} \mathrm{dNTP}(2.5 \mathrm{mM})$, and $0.5 \mu \mathrm{l}$ Taq Polymerase (5 U/ $/ \mu \mathrm{l})$ (Takara Biotechnology Co., Ltd., Dalian, China). The PCR reactions were carried out as follows: 1 cycle of $94^{\circ} \mathrm{C}$ for $5 \mathrm{~min}, 30$ cycles of $94^{\circ} \mathrm{C}$ for $45 \mathrm{sec}, 57^{\circ} \mathrm{C}$ for $45 \mathrm{sec}$ and $72^{\circ} \mathrm{C}$ for $1 \mathrm{~min}$, followed by 1 cycle of $72^{\circ} \mathrm{C}$ for $7 \mathrm{~min}$. The PCR product was digested with $A l u \mathrm{I}$ restriction endonuclease (Takara Biotechnology Co., Ltd, Otsu, Shiga) overnight at $37^{\circ} \mathrm{C}$. The digest was loaded onto a $2 \%$ agarose gel and run at $80 \mathrm{~V}$ for $40 \mathrm{~min}$, then visualized under ultraviolet light staining with ethidium bromide. A 50-base pair (bp) DNA ladder (Takara Biotechnology) was used to assess DNA size. The 366-bp PCR product had a consistent restriction site, resulting in a 98 - and a 268 -bp fragment. The GG genotype did not have a second cutting site for AluI. The CC genotype had a second cutting site resulting in 3 fragments of 205, 98 and 63 bp in size. The heterozygous GC genotype was characterized by 4 bands of 268, 205, 98 and $63 \mathrm{bp}$.

Enzyme-linked immunosorbent assay (ELISA) used for the detection of serum concentrations of MIF. Blood samples were collected from 102 patients with cervical cancer (comprising 21 patients with and 81 without lymphatic metastasis) and 48 healthy controls. Collected EDTA-anticoagulated peripheral blood samples $(5 \mathrm{ml})$ were centrifuged at $3000 \mathrm{rpm}$ for 5 min. Supernatants of anticoagulated plasma were collected and stored at $-70^{\circ} \mathrm{C}$ until use. The ELISA kit was provided by R\&B Systems (Minneapolis, MN, USA). ELISA was performed according to the manufacturer's instructions and repeated twice to obtain the average. Absorbance at $492 \mathrm{~nm}$ was tested using a spectrophotometer.

Statistical analyses. Differences in the distributions of genotypes and allele frequencies of the MIF-173G/C variants in various groups were evaluated using the $\chi^{2}$ test. The associations between MIF genotypes and risk of cervical cancer were estimated by computing the odds ratios (OR) and their 95\% confidence intervals (95\% CI). The HardyWeinberg equilibrium was tested using a goodness-of-fit $\chi^{2}$ test to compare the observed genotype frequencies with those expected among the control individuals. MIF serum concentrations were expressed as $\overline{\mathrm{x}} \pm \mathrm{s}$. The F-value was calculated and the differences in serum concentrations between the various groups were compared. Statistical significance was considered for two-tailed P-values of $<0.05$. Statistical analyses were performed using SPSS 13.0.

\section{Results}

Selected characteristics of the 250 cervical cancer cases and 147 cancer-free controls are shown in Table I. Of the 250 cervical cancer cases, $234(93.6 \%)$ were squamous cell carcinoma, $6(2.4 \%)$ were adenocarcinoma and $2(0.8 \%)$ were adenosquamous carcinoma. With regard to tumor stage, $23(9.2 \%)$ cases were stage I carcinoma, 139 (55.6\%) were stage II, $72(28.8 \%)$ were stage III and $16(6.4 \%)$ had 
Table I. Demographic and selected variables in cervical cancer patients and controls.

\begin{tabular}{lccc}
\hline Variables & $\begin{array}{c}\text { Cases } \\
(\mathrm{n}=250)\end{array}$ & $\begin{array}{c}\text { Controls } \\
(\mathrm{n}=147) \\
\mathrm{n}(\%)\end{array}$ & P-value \\
\hline Age (years; & $49.08 \pm 9.405$ & $47.99 \pm 10.750$ & 0.639 \\
mean \pm SD) & & & \\
Parity & & & 0.023 \\
0-1 & $34(13.6)$ & $30(20.0)$ & \\
2 & $96(38.4)$ & $62(42.3)$ & \\
>2 & $120(48.0)$ & $55(37.7)$ & \\
Family history & & & \\
of cancer & & & \\
No & $212(84.8)$ & $137(93.2)$ & \\
Yes & $38(15.2)$ & $10(6.8)$ & \\
Histological type & & & \\
Squamous cell & $234(93.6)$ & & \\
$\quad$ carcinoma & & & \\
Adenocarcinoma & $6(2.4)$ & & \\
Adenosquamous & $2(0.8)$ & & \\
Others & $8(3.2)$ & & \\
Stage & & & \\
I & & & \\
II & & & \\
III & & & \\
Unknown & & & \\
Contact bleeding & & & \\
No & & & \\
Yes & & & \\
Lymphatic & & & \\
metastasis & & & \\
No & & & \\
Yes & & & \\
\hline
\end{tabular}

$\mathrm{SD}$, standard deviation.

no staging information. In total, $84(33.6 \%)$ patients exhibited contact bleeding, and 166 (66.4\%) did not. Compared with the control subjects, the cervical cancer cases had a significantly higher parity $(\mathrm{P}=0.023)$, and higher family history of any types of cancer $(\mathrm{P}=0.009)$.
The Hardy-Weinberg equilibrium was maintained in MIF-173G/C in the blood of the cervical cancer and healthy control groups, indicating that this polymporphism was present in each of the two groups (Table II).

Allele and genotype frequencies were measured in 250 patients and 147 controls for this biological polymorphism. Significantly different genotype distributions were found between cervical cancer patients and healthy controls $(\mathrm{P}=0.022)$. In particular, the incidence of the MIF wild-type genotype $(\mathrm{GG})$ in patients was lower $(16.8 \%)$ than that in the controls (27.21\%). The distribution of mutant genotypes (GC and $\mathrm{CC}$ ) was higher among patients $(83.20 \%)$ than the controls $(72.79 \%)$. A statistically significant risk $(\mathrm{OR}=1.851, \mathrm{P}=0.013)$ was observed for the combined $\mathrm{CC}$ and $\mathrm{GC}$ genotypes. These mutant genotypes were identified as possible causative factors increasing the risk of primary cervical cancer. The same results were subsequently observed in the groups with and without lymphatic metastasis, as well as in the various differentiation groups. The carriers of the GC and CC genotypes exhibited a higher risk of lymphatic metastasis 1.666 times that of $\mathrm{GG}$ genotype carriers $(\mathrm{P}=0.019)$. Furthermore, $\mathrm{C}$ allele carriers exhibited a risk of lymphatic metastasis 1.850 times that of $\mathrm{G}$ allele carriers $(\mathrm{P}=0.012)$. These results indicate that the mutant genotypes of $\mathrm{GC}$ and $\mathrm{CC}$ and $\mathrm{C}$ allele may play a significant role in the development of cervical cancer and lymphatic metastasis (Table III).

The ELISA revealed that that the average serum concentrations of MIF were statistically significantly different between cervical cancer patients and healthy controls, as well as in the groups with and without lymphatic metastasis and between the groups presenting various degrees of tumor differentiation $(\mathrm{P}<0.05)$ (Table IV).

Combined effects of MIF polymorphism and serum MIF concentration for cervical cancer. Tables V-VII show the effects of MIF polymorphism and serum MIF concentration for cervical cancer. The serum MIF concentration was higher in patients than in controls across each genotype $(\mathrm{P}<0.05)$. However, GC or CC genotype carriers exhibited higher levels of MIF expression than patients without those genotypes. The lymphatic metastasis group exhibited a higher MIF serum expression than the group without lymphatic metastasis. Additionally, in the lymph metastasis group, GC or CC genotype carriers exhibited higher levels of MIF serum expression than patients without those genotypes. Serum MIF concentrations were higher in the poorly differentiated group

Table II. Hardy-Weinberg equilibrium examination of the MIF-173G/C genotypes in Shanxi province

\begin{tabular}{|c|c|c|c|c|c|c|c|c|}
\hline \multirow[b]{2}{*}{ Genotype } & \multicolumn{4}{|c|}{ Case group } & \multicolumn{4}{|c|}{ Control group } \\
\hline & Practiced & Expected & Overall & HWE & Practiced & Expected & Overall & HWE \\
\hline GG & 42 & 40.4 & 250 & $\mathrm{P}>0.05$ & 40 & 37.3 & 147 & $P>0.05$ \\
\hline GC & 117 & 120.2 & & & 68 & 73.4 & & \\
\hline $\mathrm{CC}$ & 91 & 89.4 & & & 39 & 36.3 & & \\
\hline
\end{tabular}

HWE, Hardy-Weinberg equilibrium. 





Table IV. MIF serum concentrations.

\begin{tabular}{lrrr}
\hline Group & $\mathrm{N}$ & $\overline{\mathrm{x}} \pm \mathrm{s}(\mathrm{ng} / \mathrm{ml})$ & $\mathrm{P}$-value \\
\hline Controls & 48 & $11.24 \pm 1.86$ & $0.000^{\mathrm{a}}$ \\
Patients & 102 & $16.31 \pm 5.07$ & $0.002^{\mathrm{b}}$ \\
Lymphatic metastasis & 21 & $23.88 \pm 5.03$ & $0.000^{\mathrm{c}}$ \\
No lymphatic metastasis & 81 & $14.34 \pm 2.69$ & $0.000^{\mathrm{e}}$ \\
Well-differentiated & 19 & $11.40 \pm 1.70$ & $0.000^{\mathrm{f}}$ \\
Moderately differentiated & 57 & $16.30 \pm 3.48$ & $0.000^{\mathrm{g}}$ \\
Poorly differentiated & 26 & $19.92 \pm 6.55$ & \\
\hline
\end{tabular}

Statistically significant difference between average MIF concentrations in ${ }^{\mathrm{a}}$ controls and patients; ${ }^{\mathrm{b}}$ controls and patients with lymphatic metastasis; ${ }^{c}$ controls and patients without lymphatic metastasis; ${ }^{\mathrm{p}}$ patients with and without lymphatic metastasis; ${ }^{\mathrm{e}}$ well and moderately differentiated groups; ${ }^{\mathrm{f}} \mathrm{well}$ and poorly differentiated groups; and ${ }^{\mathrm{g}}$ moderately and poorly differentiated groups; $(\mathrm{P}<0.05)$, for all concentrations .

Table V. Serum concentrations in controls and early cervical cancer patients with three genotypes.

\begin{tabular}{|c|c|c|c|c|c|c|c|}
\hline \multirow{2}{*}{ Group } & & \multirow{2}{*}{$\begin{array}{l}\mathrm{n} \\
\mathrm{GG}\end{array}$} & \multicolumn{2}{|l|}{ Genotype (ng/ml) } & & \multirow{2}{*}{ F-value } & \multirow{2}{*}{ P-value } \\
\hline & & & GC & $\mathrm{CC}$ & & & \\
\hline Control & 48 & $8.535 \pm 1.633$ & $13.655 \pm 0.530$ & $11.441 \pm 1.500$ & $11.314 \pm 2.028$ & 6.386 & $0.019^{\mathrm{a}}$ \\
\hline \multirow[t]{2}{*}{ Patient } & 102 & $13.574 \pm 3.144$ & $18.689 \pm 4.817$ & $21.260 \pm 7.013$ & $17.570 \pm 5.919$ & 7.212 & $0.002^{\mathrm{b}}$ \\
\hline & & $12.981 \pm 3.408$ & $18.060 \pm 4.805$ & $17.332 \pm 7.325$ & $16.156 \pm 5.898$ & 4.221 & $0.020^{\mathrm{c}}$ \\
\hline
\end{tabular}

Statistically significant difference between average MIF concentrations of 3 genotypes in the ${ }^{\mathrm{a}}$ control group; ${ }^{\mathrm{b}}$ patient group, and ${ }^{\mathrm{c}} \mathrm{between}$ the control and patient groups; $(\mathrm{P}<0.05)$, for all concentrations.

than in the well-differentiated one. These results indicate that, as GC or CC genotype carriers exhibited higher serum MIF concentrations, which increase the risk of cervical cancer and subsequent lymph metastasis, the degree of differentiation of tumor cells may be poorer than that of GG genotype carriers.

\section{Discussion}

This study investigated the association between cervical cancer and functionally active polymorphisms in the MIF gene. A marked correlation exists between MIF gene polymorphism and human autoimmune and inflammatory diseases. Donn et al first found that the MIF gene exhibits an SNP dot in the promoter region, a mutation from $\mathrm{G}$ to $\mathrm{C}$. Previous studies have shown that there are at least 200 genetic polymorphisms in the MIF gene, which are concentrated in four areas: the promoter regions -794 CATT repeat sequences, 5' flank area $-173 \mathrm{G} \rightarrow \mathrm{C}$ mutations, and the intron regional +254 $(\mathrm{T} / \mathrm{C})$ and $+656(\mathrm{C} / \mathrm{G})(19)$. Intron area gene polymorphism is not considered to be significant. However, the polymorphisms of the promoter and explicit area affect transcription activity and disease susceptibility. Among them, the polymorphism in the promoter region may alter the expression of mRNA, further adjusting the expression of the protein. MIF-173G/C increases susceptibility to certain diseases. Arisawa et al (15) found that the $\mathrm{C}$ allele is a risk factor for gastric carcinogenesis. Meyer-Siegler et al (16) found that the incidence of prostate cancer was significantly increased among GC and CC genotype carriers. Xue et al (20) found that the MIF-173G/C polymorphism was an etiological factor in childhood acute lymphoblastic leukemia and identified it as a potential candidate gene for assessing susceptibility to this malignancy.

This study adopted a restriction fragment length polymorphism polymerase chain reaction to analyze the genotype of patients and healthy controls. In the case of the -173 allele, there was a trend towards underrepresentation of the GG genotype among the cervical cancer patients. However, this relationship did not achieve statistical significance $(\mathrm{P}=0.065)$. With regard to the frequencies of the $\mathrm{CC}$ and $\mathrm{GC}$ genotypes and alleles, these polymorphisms were more frequent among the cervical cancer patient, lymph metastasis, and poorly differentiated groups than in the control, no metastasis and well-differentiated groups, respectively. $(\mathrm{p}<0.05)$. This observation indicates that the polymorphism of MIF-173G/C may correlate with the incidence of cervical cancer, risk of lymph node metastasis and malignant degree. This is consistent with results obtained by Donn et al (19). The GC and CC genotypes and $\mathrm{C}$ allele are risk factors in early-stage cervical cancer. Compared to GG genotype carriers, the risk of lymph node metastasis may be higher and the malignant degree may be more serious, potentially resulting in poorer prognosis.

A number of cytokine gene polymorphisms have been associated with altered levels of cytokine production or activity, susceptibility to various diseases (21-22), and tumor initiation, growth and metastasis (23). MIF is a cell factor stem from activated $\mathrm{T}$ lymphocytes and macrophages, which plays 
Table VI. Serum concentration in the groups with and without lymphatic metastasis with three genotypes.

\begin{tabular}{lccccccc}
\hline \multirow{2}{*}{ Group } & & \multicolumn{3}{c}{ Genotype $(\mathrm{ng} / \mathrm{ml})$} & & F-value & P-value \\
\cline { 3 - 7 } & $\mathrm{n}$ & & $\mathrm{GG}$ & $\mathrm{GC}$ & $\mathrm{CC}$ & & \\
No lymphatic metastasis & 81 & $12.185 \pm 2.285$ & $16.277 \pm 1.027$ & $15.058 \pm 2.815$ & $14.265 \pm 2.743$ & 8.667 & $0.002^{\mathrm{a}}$ \\
Lymphatic metastasis & 21 & $17.393 \pm 1.482$ & $23.032 \pm 6.052$ & $27.462 \pm 2.284$ & $23.300 \pm 5.586$ & 6.154 & $0.016^{\mathrm{b}}$ \\
& & 13.574 & 18.690 & 21.260 & 17.570 & 7.212 & $0.002^{\mathrm{c}}$ \\
\hline
\end{tabular}

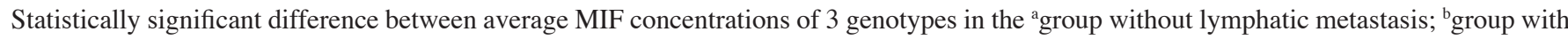
lymphatic metastasis; 'between the groups with and without lymphatic metastasis; $(\mathrm{P}<0.05)$, for all concentrations.

Table VII. MIF serum concentration in differentiation groups with three genotypes.

\begin{tabular}{|c|c|c|c|c|c|c|}
\hline \multirow{2}{*}{ Group } & \multirow{2}{*}{$\mathrm{n}$} & \multicolumn{2}{|r|}{ Genotype (ng/ml) } & \multirow[b]{2}{*}{$\mathrm{CC}$} & \multirow{2}{*}{ F-value } & \multirow{2}{*}{ P-value } \\
\hline & & GG & GC & & & \\
\hline Well differentiated & 19 & $9.220 \pm 1.213$ & $11.341 \pm 0.751$ & $10.762 \pm 1.124$ & 20.474 & $0.000^{\mathrm{a}}$ \\
\hline Moderately differentiated & 57 & $13.641 \pm 1.019$ & $17.482 \pm 0.987$ & $22.911 \pm 1.121$ & 554.769 & $0.000^{\mathrm{b}}$ \\
\hline Poorly differentiated & 26 & $15.711 \pm 0.876$ & $17.981 \pm 1.051$ & $19.264 \pm 1.219$ & 4.625 & $0.013^{\mathrm{c}}$ \\
\hline
\end{tabular}

Statistically significant difference between average MIF concentrations of 3 genotypes in the a well-differentiated group; ${ }^{\mathrm{b}}$ moderately differentiated group; and 'poorly differentiated group; $(\mathrm{P}<0.05)$, for all concentrations.

a crucial role in the inflammation and immunoreactions. MIF is also a significant factor in the development of tumors, and is involved in the proliferation and differentiation of tumor cells, the formation of tumor blood vessels, and the inhibition of tumor cell apoptosis, with a high concentration observed in certain pre-cancerous diseases (24). Our study used ELISA to examine the serum concentrations of MIF in early stage cervical cancer patients and healthy controls, as well as groups of cervical cancer patients with and without lymph node metastasis and three groups with different degrees of differentiation. Our results demonstrate that the serum concentrations of MIF in the early cervical cancer, lymph node metastasis and low differentiation groups exhibited a rising trend. Our study results showed that concentrations in healthy individuals are $<12 \mathrm{ng} / \mathrm{ml}$ : the risk of early cervical cancer increases with concentrations $>12 \mathrm{ng} / \mathrm{ml}$; incidence of lymph node metastasis is markedly increased with concentrations of $>15 \mathrm{ng} / \mathrm{ml}$; and concentrations $>11.4 \mathrm{ng} / \mathrm{ml}$ are correlated with lower degrees of tumor differentiation, higher degrees of malignancy and poorer prognosis. Serum concentrations of MIF are therefore capable of being used to predict early cervical cancer, assess the risk of lymph node metastasis and degree of tumor malignancy, and provide a beneficial diagnostic and prognostic tool for clinical application (Table IV).

In combination with MIF serum concentrations and MIF genetic polymorphism, MIF-173 promoter region SNPs affect MIF gene expression and thus have an impact on serum MIF levels. Statistically significant differences were observed between the average blood serum concentrations of MIF in the patient and control groups $(\mathrm{F}=4.221, \mathrm{P}=0.020)$; the groups with and without lymph node metastasis $(\mathrm{F}=7.212, \mathrm{P}=0.002)$; and the three groups with various degrees of differentiation
$(\mathrm{P}<0.05)$. GC or $\mathrm{CC}$ genotype or $\mathrm{C}$ allele carriers exhibit high levels of MIF in serum, and are more likely to develop early cervical cancer, lymph node metastasis and higher degrees of tumor malignancy. However, MIF serum concentrations $>13 \mathrm{ng} / \mathrm{ml}$ were associated with increased risk of early cervical cancer, and levels $>14 \mathrm{ng} / \mathrm{ml}$ were associated with increased risk of lymph node metastasis even in GC (wildtype) carriers.

The present study had certain limitations. The study was hospital-based and was conducted in the region of the Shanxi Province, China. Therefore, it was not possible to eliminate selection bias. Furthermore, since the current study did not include all clustered polymorphism sites of MIF and associated genes, various types of analysis could not be carried out. Therefore, further large-scale population-based studies should be conducted, including the analysis of various clustered polymorphisms.

In conclusion, monitoring serum concentrations of MIF and evaluating the MIF-173 dot genotype may aid in the assessment of the risks of early cervical cancer, lymph node metastasis and degree of malignancy in the clinic. It may also benefit prognosis and screening of the high-risk population of early cervical cancer, and provide a significant clinical marker for the prevention and further treatment of early cervical cancer.

\section{Acknowledgements}

This study was supported in part by the Department of Biochemistry and Molecular Biology, Shanxi Medical University, Taiyuan, China, Department of Gynecology, Shanxi Province Tumor Hospital, Taiyuan, China. 


\section{References}

1. Parkin DM, Bray F, Ferlay J and Pisani P: Global cancer statistics, 2002. CA Cancer J Clin 55: 74-108, 2005.

2. Ito T, Ishizuka T, Suzuki K, et al: Cervical cancer in young Japanese women. Arch Gynecol Obstet 264: 68-70, 2000.

3. Chan PG, Sung HY and Sawaya GF: Changes in cervical cancer incidence after three decades of screening US women less than 30 years old. Obstet Gynecol 102: 765-773, 2003.

4. Wen C: China's plans to curb cervical cancer. Lancet Oncol 6: 139-141, 2005.

5. Brandao VCRAB, Lacerda HR, Lucena-Silva $N$ and Ximenes RA: Frequency and types of human papillomavirus among pregnant and non-pregnant women with human immunodeficiency virus infection in Recife determined by genotyping. Mem Inst Oswaldo Cruz 104: 755-763, 2009.

6. Munoz N, Bosch FX, de Sanjose S, et al: Epidemiologic classification of human papillomavirus types associated with cervical cancer. N Engl J Med 348: 518-527, 2003.

7. Coussens LM and Werb Z: Inflammation and cancer. Nature 420: $860-867,2002$.

8. Cordon-Cardo $\mathrm{C}$ and Prives C: At the crossroads of inflammation and tumorigenesis. J Exp Med 190: 1367-1370, 1999.

9. Karin M, Lawrence T and Nizet V: Innate immunity gone awry: linking microbial infections to chronic inflammation and cancer. Cell 124: 823-835, 2006.

10. Conroy H, Mawhinney L and Donnelly SC: Inflammation and cancer: macrophage migration inhibitory factor (MIF) - the potential missing link. QJM 103: 831-836, 2010.

11. Rendon BE, Willer SS, Zundel W and Mitchell RA: Mechanisms of macrophage migration inhibitory factor (MIF)-dependent tumor microenvironmental adaptation. Exp Mol Pathol 86: 180-185, 2009.

12. Cooke G, Armstrong ME and Donnelly SC: Macrophage migration inhibitory factor (MIF), enzymatic activity and the inflammatory response. Biofactors 35: 165-168, 2009.

13. Hamatake M, Yoshino I, Tomiyasu M, et al: Intratumoral expression of macrophage migration inhibitory factor is correlated with serum C-reactive protein and interleukin-6 in patients with non-small cell lung cancer. Surg Today 38: 921-925, 2008
14. Xu X, Wang B, Ye C, et al: Overexpression of macrophage migration inhibitory factor induces angiogenesis in human breast cancer. Cancer Lett 261: 147-157, 2008.

15. Arisawa T, Tahara T, Shibata T, et al: Functional promoter polymorphisms of the macrophage migration inhibitory factor gene in gastric carcinogenesis. Oncol Rep 19: 223-228, 2008.

16. Meyer-Siegler KL, Vera PL, Iczkowski KA, et al: Macrophage migration inhibitory factor (MIF) gene polymorphisms are associated with increased prostate cancer incidence. Genes Immun 8: 646-652, 2007.

17. Paralkar V and Wistow G: Cloning the human gene for macrophage migration inhibitory factor (MIF). Genomics 19: 48-51, 1994.

18. Donn RP, Shelley E, Ollier WE and Thomson W: A novel 5 -flanking region polymorphism of macrophage migration inhibitory factor is associated with systemic-onset juvenile idiopathic arthritis. Arthritis Rheum 44: 1782-1785, 2001.

19. Donn R, Alourfi Z, de Benedetti F, et al: Mutation screening of the macrophage migration inhibitory factor gene: positive association of a functional polymorphism of macrophage migration inhibitory factor with juvenile idiopathic arthritis. Arthritis Rheum 46: 2402-2409, 2002.

20. Xue Y, Xu H, Rong L, et al: The MIF -173G/C polymorphism and risk of childhood acute lymphoblastic leukemia in a Chinese population. Leuk Res 34: 1282-1286, 2010.

21. Martinez A, Orozco G, Varade J, et al: Macrophage migration inhibitory factor gene: influence on rheumatoid arthritis susceptibility. Hum Immunol 68: 744-747, 2007.

22. Wu J, Chen F, Zhang X, et al: Association of MIF promoter polymorphisms with psoriasis in a Han population in northeastern China. J Dermatol Sci 53: 212-215, 2009.

23. McClelland $M$, Zhao L, Carskadon S and Arenberg D: Expression of CD74, the receptor for macrophage migration inhibitory factor, in non-small cell lung cancer. Am J Pathol 174: 638-646, 2009

24. Bach JP, Rinn B, Meyer B, Dodel R and Bacher M: Role of MIF in inflammation and tumorigenesis. Oncology 75: 127-133, 2008. 